\title{
Recursos y obstáculos para la autorrealización de estudiantes de colegios nocturnos costarricenses
}

\author{
Irma Arguedas Negrini \\ Maestra en Orientación Escolar \\ Docente, Escuela de Orientación y Educación Especial \\ Facultad de Educación, Universidad de Costa Rica, Costa Rica \\ irma.arguedas@ucr.ac.cr
}

Recepción: 25-06-2019 / Aceptación: 07-10-2019

\section{Resumen}

El estudio cualitativo tuvo como propósito analizar los factores que conducen a que personas adultas jóvenes consideren sus vidas autorrealizadas, a fin de dirigir esfuerzos en la Orientación hacia la promoción del desarrollo integral en esa etapa de la vida. Para ello se realizó la descripción de recursos

y obstáculos en la construcción de dichos factores; se aplicó el método fenomenológico y se utilizó el análisis temático.

La investigación se llevó a cabo con veintiún estudiantes de dos colegios nocturnos de Costa Rica, mediante entrevistas individuales y grupos focales.

Palabras clave: Orientación, autorrealización, educación nocturna, desarrollo integral, fortalezas, obstáculos.

\begin{abstract}
The qualitative study had the purpose to analyze the factors that lead young adults to consider their lives self-realizing, in order to direct efforts in the Orientation towards the promotion of integral development at that stage of life. For this, the description of resources and obstacles in the construction of mentioned factors was made; The phenomenological method was applied and thematic analysis was used.
\end{abstract}

The research was carried out with twenty-one students from two night schools in Costa Rica, through individual interviews and focus groups.

Keywords: Orientation, self-realization, night education, integral development, strengths, obstacles. 


\section{Introducción}

En este trabajo se reportan los hallazgos de una investigación que se implementó desde la perspectiva de la Orientación, con el objetivo de aportar insumos para la promoción del desarrollo integral de personas adultas jóvenes. Esto en concordancia con lo planteado por González (2011) y Jiménez (2015), en cuanto a que la función educativa de esta disciplina se dirige a la potenciación de recursos de individuos y grupos, a lo largo de las diferentes etapas. Se indagó acerca de tres aspectos relacionados con las trayectorias vitales de las personas participantes: lo que les hace sentirse autorrealizadas, los recursos con que han contado y los obstáculos que han enfrentado; ya que las adversidades también son componentes de los procesos de desarrollo humano (Hill y Buss, 2008).

La pregunta: “¿Qué voy a hacer de mi vida?”, que se remite más a población adolescente, es pertinente en todas las etapas del ciclo vital, cuando se vivencian cambios en la condición de salud, el empleo o estudio y las relaciones interpersonales, entre otros (Savickas et al., 2009). En estos procesos, la utilización de las propias fortalezas es esencial.

En las intervenciones de Orientación, las fortalezas deben explicitarse para que así sea posible su edificación y aplicación en situaciones concretas, con miras a su incorporación permanente al repertorio de las personas (Wong, 2015). Los seres humanos necesitan a lo largo de sus vidas contar con la libertad para dirigirse hacia lo que les permite utilizar sus ca- pacidades, fortalezas e intereses (Southwick y Charney, 2012).

La autorrealización guarda una estrecha relación con las fortalezas humanas, debido a que consiste en concretar la expresión de las propias capacidades, en función de aspiraciones y metas para el provecho, no solo de la persona a nivel individual, sino también en su dimensión social (Gewirth, 1998). Una característica esencial de las fortalezas es que se pueden modificar y cultivar. Para BernalGuerrero (2002), autorrealización es integración de metas y completud en la superación de dificultades.

Es interesante anotar que las metas extrínsecas, el reconocimiento social, el éxito financiero y el atractivo físico no se relacionan con altos niveles de bienestar, como sí lo hacen las metas de intimidad, espiritualidad y generatividad.

Las aspiraciones motivadas por la intimidad son aquellas dirigidas al establecimiento de relaciones profundas y mutuamente gratificantes; la generatividad se refiere a la entrega a las demás personas y la influencia en generaciones más jóvenes; las aspiraciones de tipo espiritual son aquellas relacionadas con propósitos ulteriores, ética y el contacto con lo sublime en la experiencia cotidiana. Cabe aclarar que si bien Erikson (1971) planteaba que la intimidad y la generatividad tienen edades o periodos en que se enfatizan, actualmente se consideran motivaciones que no tienen especificidad etaria (Emmons, 2003). 
Adicionalmente, diversas investigaciones han evidenciado que el interés social se relaciona tanto con el significado y equilibrio vitales, como con mejores estados de salud física y mental. Más aún, tener objetivos claros y compromiso con una misión, proporciona fuerza y coraje. La valoración por parte de las personas de su trabajo y las actividades a las que se dedica, igualmente contribuye con el manejo del estrés o crisis que se presentan en la comunidad, escuela o trabajo (Miller y Taylor, 2016).

En este estudio, la indagación sobre metas y recursos del estudiantado participante se basó en las siguientes dimensiones del bienestar descritas por con Ryff (2013):

- Auto-aceptación: El polo positivo se caracteriza por la presencia de actitudes positivas hacia uno mismo y la propia historia, y por tener una visión favorable de la misma. Las personas más cercanas al polo negativo se sienten insatisfechas y decepcionadas.

\section{- Relaciones positivas con otras personas:}

El polo positivo se refleja en relaciones cálidas y de confianza; preocupación por el bienestar de los demás, capacidad de empatía, afecto y correspondencia en las relaciones. El polo negativo de esta dimensión se manifiesta en pocas relaciones estrechas, predominio de relaciones que conllevan frustración, así como dificultad para sentir calidez y preocupación hacia otros.

- Autonomía: La presencia de regulación interna, de resistencia a presiones sociales para pensar y actuar de formas determinadas, caracteriza al polo positivo de esta dimensión. Lo que caracteriza al polo negativo de la dimensión de autonomía es la excesiva preocupación por las evaluaciones y expectativas de las demás personas.

- Dominio de variables del entorno: La persona más cercana al polo positivo es competente en el manejo de los contextos en que se desenvuelve; hace uso efectivo de oportunidades y puede crear o elegir los ambientes más acordes con sus necesidades y valores. La persona más inclinada hacia el polo negativo tiene dificultades en el manejo de asuntos cotidianos, desconoce las oportunidades que tiene a su alcance y carece de control sobre el mundo externo.

- Propósito de vida: El tener metas y encontrar un sentido a la vida presente y pasada son aspectos del polo positivo. Por el contrario, carecer de direccionalidad, no encontrar un sentido a las experiencias pasadas, ni tener las creencias que favorecen el descubrimiento de un sentido de vida, reflejan el polo negativo. Además de lo que plantea Ryff (2013) en sus dimensiones del bienestar, variedad de autores (Emmons, 2003; Fiorentino, 2008; Frankl, 2012; Webb, 2013) han reconocido la participación en proyectos y actividades que van más allá del interés, estrictamente personal, como un factor positivo para el desarrollo.

- Crecimiento personal: En el polo positivo se encuentran la sensación de una mejora continua, realización del propio potencial y 
desarrollo de la efectividad. En el polo negativo se encuentran la sensación de estancamiento, el aburrimiento y la dificultad para cambiar actitudes o comportamientos.

\section{Metodología}

Se eligió el enfoque cualitativo debido al interés por profundizar en las perspectivas de las personas participantes. El método utilizado fue la fenomenología, por centrarse en las formas en que las personas experimentan y dan significado a diversas vivencias, mediante la descripción de situaciones específicas y clarificando los significados que surgen (Kvale, 2011).

Para el estudio se optó por abordar a estudiantes de colegios nocturnos, ya que -además de encontrarse en la etapa de la adultez joven- estos han estado en condiciones de desventaja en comparación con otras modalidades de educación secundaria.

Tras la autorización de las Direcciones de dos colegios de educación secundaria en Costa Rica: Sección nocturna del Colegio Técnico Profesional de Calle Blancos y Colegio Nocturno la Unión, dentro de cada una de ellos se extendió la invitación de participación a tres hombres y tres mujeres. Los participantes debían cumplir los siguientes requisitos: ser mayores de edad, y que en el año 2017 no estuvieran cursando el último nivel. Esto con la finalidad de contactarles por medio de las orientadoras al año siguiente, en caso de requerir entrevistas adicionales. Asimismo, y para posteriormente dar mayor robustez al análisis, se extendió la invitación para fungir como informantes en un grupo focal a nueve estudiantes con las mismas características.

Las técnicas utilizadas fueron la entrevista semi-estructurada y el grupo focal. La investigación partió de las metas de las personas participantes, tomando como base las dimensiones del bienestar de Ryff (2013). La guía de entrevista y la conducción del grupo focal fueron validadas por dos personas del mismo grupo etario invitado a participar (Rodríguez, Gil y García, 1999).

Para diez participantes, las entrevistas individuales tuvieron dos etapas (entrevista inicial y de verificación). Pero para dos personas de la muestra, estas entrevistas se realizaron en un único momento, debido a que uno de ellos salió del sistema educativo en el transcurso de la vigencia del proyecto; y para el otro fue imposible asistir a las citas concertadas por razones de horario laboral.

El grupo focal se llevó a cabo con estudiantes de la sección nocturna del Colegio Técnico Profesional de Calle Blancos, Costa Rica. Cada encuentro fue grabado con el consentimiento de las personas participantes, y luego transcrito textualmente.

El estudio recibió el apoyo de la Escuela de Orientación y Educación Especial, el Instituto de Investigación en Educación (INIE) y la Vicerrectoría de Investigación de la Universidad de Costa Rica (Proyecto No 724-B7-304). 


\section{Resultados}

Para procesar los datos se siguieron los lineamientos de Maguire y Delahunt (2017) para el análisis temático. Esta técnica permite identificar patrones o temas dentro de un cuerpo de información. Un tema captura aspectos de los datos que son relevantes para la pregunta de investigación y representa un patrón en las respuestas o significados.

Los temas o patrones dentro de los datos se identifican tanto de forma inductiva (que parte de los datos recolectados) como deductiva (que parte de la teoría). La técnica también permite la identificación de subtemas.

El análisis de la información recolectada arrojó los temas que se muestran en la figura 1 .

A continuación, se presentan los temas y subtemas, sustentados en expresiones textuales de los participantes:

\section{a. Propósitos}

Además de las metas educativas, se han propuesto superar adversidades, mediante la identificación de significados y aprendizajes adquiridos en las vivencias difíciles.

- Seguir estudiando. Se enfocan en concluir la secundaria o su formación técnica e idealmente cursar otros estudios. Sobresale el deseo de ayudar a otras personas y de mejorar la calidad de vida propia y colectiva, como motivación para alcanzar sus metas educativas:

"No me quiero estancar, quiero seguirme superando (...). Sacar profesión (...) Ayudar a la familia a salir de la pobreza” (Juan).

"Tratar de salir adelante con mis hijos (...) lo que deseo es tener ese título de bachillerato en mis manos (...), el sacar el bachi es un reto que, nos ponemos desde que empezamos,

\begin{tabular}{|l|c|}
\hline \multicolumn{1}{|c|}{ Temas } & $\begin{array}{c}\text { Aspecto indagado } \\
\text { al que responde }\end{array}$ \\
\hline a. Propósitos & $\begin{array}{c}\text { Fuentes de } \\
\text { autorrealización }\end{array}$ \\
\hline b. Fortalezas internas: & \\
- Valores & \\
- Cogniciones optimistas & \\
- Acciones concretas para el enriquecimiento de relaciones interpersonales & Recursos \\
- Estilo educativo & \\
c. Fortalezas externas: & \\
- Apoyos brindados por la institución educativa & \\
- Apoyos brindados por personas significativas & \\
\hline d. Limitaciones & Obstáculos \\
\hline
\end{tabular}

Figura 1. Temas de autorrealización del estudiantado. 
desde séptimo. Trabajar para darles una vida digna y una mejor calidad de vida" (Daniela).

- Superación de adversidades. Se han esforzado por salir adelante y por aprender de las experiencias. Como puede notarse en las citas textuales, el componente de contribuir con otras personas de nuevo se manifiesta:

"Para uno es muy duro (...) igual en el corazón queda, pero nunca hay que dejarse vencer" (Jordan).

"Mientras hay vida hay esperanza (...) Si cometió un error (...) lo importante es la actitud, el espíritu de superación que te hace seguir adelante (...) Hay cosas que yo he visto que no hay salida, pero a lo último eso me ayudó a que yo entendiera algo, y ese aprendizaje me ayudó para otras cosas que estoy superando" (Priscila).

Al indagar con una chica llamada María, acerca de lo que recomendaría a otras personas, con base en sus vivencias, indica: "Nunca dejes de ser feliz, sigue luchando por tus sueños".

El esfuerzo también se dirige hacia aprender a ver las cosas desde perspectivas transformadas y colmadas de sentido, que es la forma en como Wong (2012) define la espiritualidad: "Si las ve de diferente manera, cambian las cosas por más difíciles que sean" (Karina).

- Auto-trascendencia. Es dirigirse intencionalmente a algo significativo y externo a la persona (Martínez, 2013). Los participantes revelaron esta disposición al expresar, reiteradamente, la satisfacción que les da ayudar. Jordan, otro de los entrevistados, se refiere específicamente a sus hermanos: "Bueno, con mis hermanos (...). Guiarlos para que no cometan algunos errores que cometen los demás".

Las palabras de otro chico, Eduardo, sintetizan la autotrascendencia: "Aprender para el beneficio de todos. Porque al final todo en la vida es un aprendizaje (...). Como sociedad nos beneficia a todos madurar, porque al final somos una comunidad, una especie, ocupamos unirnos y dejar de pensar en cosas que beneficien a cada uno".

\section{b. Fortalezas internas}

- Valores. De acuerdo con Taff (1966), los valores son "los criterios utilizados para escoger entre alternativas al tomar decisiones o implementar acciones" ( $p$. 214). Los valores que sobresalieron son balances, autonomía, dar lo mejor de sí y responsabilidad.

- Balances. En su quehacer diario, implementan equilibrios en la distribución del tiempo y en la atención a las necesidades propias y de los demás. Por ejemplo, una chica entrevistada de nombre Karina piensa que si en un trabajo llegara a asumir una jefatura, sería respetuosa pero estricta. Por su parte, un chico llamado Guerin, ha tenido logros en expresar sus perspectivas sin recurrir a los gritos, ya que solía irse a los extremos de quedarse callado o tener expresiones explosivas; y 
ha encontrado formas de expresar sus visiones de forma respetuosa.

Otra participante de nombre Ivannia ha luchado por el balance requerido para su realización como mujer: "Mi esposo no quería que yo estudiara (...). Yo le decía yo quiero realizarme como mujer (...). Estudiar por satisfacción mía”.

Dentro del valor 'Balances' se ubica la igualdad entre personas. Juan expresó: "Yo no me doy por vencido, pero no me siento más que los demás porque todos lo podemos lograr".

- Autonomía. Quienes participaron en la investigación toman acciones por su propia convicción y no por presiones ejercidas desde fuera. Las siguientes son algunas de sus perspectivas al respecto:

"Porque todos somos diferentes (...). Lo que yo piense no puede venir una persona y cambiármelo porque eso sería una falta de respeto (...). Si tengo que decirle a alguien que opino algo diferente, lo digo" (María Fernanda).

"Yo sé que el estudiar es importante y lo hago porque yo quiero salir adelante, no porque me lo digan (...) estudiar de verdad, aprender para realizarme en el trabajo" (Karina).

A veces, la autonomía ha tenido como consecuencia la pérdida de amistades: "Diay, él llegaba a la casa casi todos los días, a hacer trabajos, aunque fuera de otra sección, o a estudiar, él me ayudaba, yo le ayudaba. Ya eso, diay, se fue perdiendo" (Guerin).
- Dar lo mejor de sí. Hubo manifestaciones repetidas en relación con el deseo de aprovechar sus potencialidades: "Sí, me gusta mucho tener buenas notas (...). Porque a mí me gusta, me gusta ser así” (Daniela).

"Cuando no estoy aquí, aprendo en el internet varias cosas como que me beneficien a la hora de ver mejor la materia. Eh, en lo personal también quiero ser mejor (...). Ser alguien respetable, que admiren y admirarme a mí mismo" (Eduardo).

- Responsabilidad. Esta se refleja en actuar sobre lo que pueden controlar, como sacar provecho de oportunidades, aceptar la propia historia y el cuido de la salud; su desarrollo personal y el trabajo. El esfuerzo continuo ha sido necesario para asumir responsabilidad, lo que no ha sido tarea fácil: "Entonces siento que me he superado de una manera $u$ otra. Y quiero seguirlo haciendo, pero a veces es duro. A veces yo, lo he dicho sinceramente, yo he querido como tirar la toalla" (Priscilla).

"Hasta los domingos tengo que trabajar. Un toque cansado (...). Yo me levanto a las tres y media y jalo en el primer bus de cuatro y quin$c e$ " (Jordan)

"Ese tema [aceptación de eventos del pasado] para mí en lo personal ha calado mucho y he tenido que trabajarlo mucho, (...). Ese trabajo ha sido constante" (Greivin).

- Cogniciones optimistas. La forma en que las personas explican los eventos que 
les suceden, que a la vez influyen en las formas de reaccionar ante ellos, se denominan estilos explicativos. Seligman (2011) ha descrito dos de estos estilos: el optimista y el pesimista.

El optimismo favorece que los eventos negativos se interpreten como superables. Por el contrario, un estilo pesimista visualiza las adversidades como permanentes, por lo que conduce a que no se busquen soluciones $y$, por consiguiente, pone a las personas en riesgo de no persistir. El persistir o continuar los esfuerzos, a pesar de la dificultad, es un ingrediente esencial en el desarrollo de los diferentes ámbitos de las personas.

Seligman, Reivich, Jaycox y Gillham (2004) enfatizan en su importancia en la preparación para enfrentar adversidades y en la edificación de herramientas para lidiar con la ansiedad, la frustración y la tristeza.

A pesar de las ventajas del optimismo, no es fácil de aplicar, ya que como menciona Seligman (2011), para incrementar las cogniciones optimistas, la persona tiene que detectar sus pensamientos pesimistas, para luego proceder a sustituirlos. Las personas participantes relatan que la mayor parte del tiempo funcionan con cogniciones optimistas, las cuales se muestran en comportamientos perseverantes y búsqueda de soluciones a los problemas.

- Perseverancia. Consiste en sostener el esfuerzo en función de metas; es más factible cuando se adopta un estilo explicativo optimista; esto debido a que la forma optimista de interpretar las situaciones conduce a mayor auto-confianza (Seligman et al., 2004).

"Agarro más el impulso para tratar de lograrlo (...). Lo que más cueste es lo que da más satisfacción"(Guerin).

"Y ahora estoy en décimo, y a miles costos porque ese es muy durísimo" (Daniela).

Las personas participantes transmiten sus cogniciones optimistas a personas cercanas. La siguiente cita de Ivannia, ilustra cómo lo hace con uno de sus hijos: "Yo le decía inténtelo, nunca se dice no puedo, hay que intentarlo, intentarlo".

\section{- Implementación de estrategias de solu-} ción. Las cogniciones optimistas facilitan que las dificultades se visualicen como características o condiciones que se pueden superar, por lo que es más posible la búsqueda de alternativas de solución.

"Si la primera oportunidad no fue como yo quise, no quiere decir que ahí voy a morir (...). Luché por ese ideal, pero van a haber obstáculos (...). A la par viene la cajita con las herramientas para que usted logre superar" (Greivin).

No todas las áreas de tu vida van a estar cien por ciento bien (... ). Ahí fue donde me empecé a espabilar. Entonces ya, que ir a citas, que estar preguntando, que aquí, que allá (Ivannia). 


\section{- Acciones concretas para el enriquecimien-} to de relaciones interpersonales. Las personas participantes construyen relaciones armónicas y fomentan climas agradables en el trabajo y otros espacios. Son varios los autores que fundamentan la importancia de las buenas relaciones interpersonales.

Desde su modelo de Orientación para el bienestar y la prevención, a lo largo del ciclo vital, Witmer y Sweeney (1992) visualizan las relaciones caracterizadas por la cooperación y el compromiso, como protectoras del estrés y generadoras de satisfacción general.

- Reciprocidad. Se refiere al respeto entre personas, a la nivelación entre dar y recibir y a la disposición de aceptar errores.

"Ponernos de acuerdo si no nos gusta algo (...). Entonces, yo le digo y ella me dice (...). Sentándonos otro rato a hablar" (María).

"Yo tengo esa característica de reciprocidad, que como yo no he tenido esa figura de familia biológica, pero Dios me ha dado una familia que es no biológica, pero demasiado grande $y$ muy buena" (Greivin).

"Si cometo un error, y tengo que disculparme, lo hago (...) acepto las cosas" (María Fernanda).

De su relación de pareja, Daniela, una participante cuenta: "Pedirle que me diga si es que algo le molesta o decirle qué me está molestando a mí (...). Pedirle responsabilidad hacia mí, respeto (...). Eso fue culpa de los dos y yo le echaba toda la culpa a él".
- Fomento de climas relacionales agradables. Las personas participantes se relacionan de forma que los ambientes en que se desenvuelven se caractericen por amabilidad, relaciones igualitarias y flexibilidad.

"Nunca me quiero sentir más que nadie"(Priscila).

"Entonces, yo trato de tratar bien a las personas, de ser respetuoso, ser amable, y que les puedo ayudar si ocupan ayuda" (Eduardo).

- Estilo educativo. Estos son los patrones de acciones y valores, que los adultos asumen con las personas menores a su cargo, al implementar comunicación y normas de comportamiento. Rabazo (1999) describió cuatro estilos, cuyas características dependen de cómo se manifiestan la afectividad y el control en las relaciones en la familia o escuela.

El estilo autoritario se caracteriza por la coerción por parte de personas adultas, lo cual conduce a comportamientos adecuados no por convicción, sino por temor al castigo. Las exigencias de madurez están por encima de las posibilidades de niños y adolescentes.

En el estilo permisivo se brinda abundante afecto, pero no se demanda responsabilidad, lo que puede implicar una pobre autorregulación.

Las personas adultas que utilizan un estilo educativo negligente se muestran indiferentes ante las necesidades y comportamientos de niños y adolescentes; sean éstos positivos o negativos. La persona adulta no 
funciona como un modelo, no proporciona afecto ni apoyo.

Ha sido demostrado que el estilo más indicado es el llamado inductivo-apoyativo. Como su nombre lo indica, induce a las personas menores a involucrarse en comportamientos constructivos, a la vez que se les ofrecen afecto y apoyos consistentes (Rabazo, 1999). Este es el estilo que más favorece la interiorización de normas, la responsabilidad, el respeto y la seguridad (Arguedas, 2010).

El estilo inductivo-apoyativo predominó en pocas de las familias de las personas participantes. Otras no crecieron bajo ese estilo educativo; sin embargo, lo han adoptado para el trato con sus hijos y sobrinos. Los subtemas correspondientes al estilo educativo son la apertura en la comunicación; la persona adulta en su rol de modelo y guía, y la complementariedad entre expectativas y apoyos.

- Comunicación abierta. Este aspecto se refiere a interaccionar de forma respetuosa y generadora de confianza. Eduardo relata cómo su padre ha abierto espacios para que reflexione acerca de lo que desea: "En eso me educó bien mi papá. Él me dijo que siempre hiciera lo que realmente quería hacer (...). No me dejo guiar por lo que los demás piensan, sino por lo que yo creo que es bueno".

A Grevin le parece importante empatizar con su hija: "Trato de comprender la visión de la niña (...). Yo no puedo pretender que mi hija se ponga a mi altura; yo tengo que ponerme a la altura de ella para poderla entender".

- Persona adulta es modelo y guía. Las personas participantes están convencidas de la importancia de establecer normas y estructura. Cuando no contaron con esa condición en su niñez, manifiestan que les hizo falta esa figura adulta que les guiara y asignara responsabilidad.

"Yo digo que no hay nada mejor que tener paz $y$ tranquilidad, y saber que uno hace las cosas bien por satisfacción. No hay que ser mediocre. Es que yo me identifico mucho porque tengo que darle un buen ejemplo a mis hijos" (Ivannia).

"Una figura paterna que me dijera 'mire, Juan, usted tiene que, cuando tenga cierta edad, siempre hacer esto y esto'. No, nunca la tuve; tuve que hacerlo yo solo" (Juan).

- Complemento entre expectativas y apoyos. Esperan el mejor esfuerzo por parte de las personas menores a su cargo, a la vez que les apoyan en sus esfuerzos por obtener logros.

[Abuelo le decía] "A echar para adelante. Siga. Si una puerta se le cerró, mil puertas se le van a abrir; pero no se quede estancado ahí donde está usted ahorita en este momento" (...). Él siempre creyó en mí" (Luis).

"Los padres tienen que apoyarlos en todo. Aunque no es alcahuetearlos; darles el apoyo, que están ahí que puedan confiar en sus padres" (Daniela). 


\section{c. Fortalezas externas}

El saber que se cuenta con respaldos durante el proceso formativo, se concibe como un elemento fundamental del desarrollo y como un pilar de la resiliencia (Henderson y Milstein, 2003; Scales y Leffert, 2004). Se encontró que durante sus trayectorias vitales, recibieron algunos apoyos significativos, y que respondieron a estos con responsabilidad. No obstante, muchas de sus necesidades quedaron insatisfechas, al no haber contado con apoyos esenciales y toparse con grandes obstáculos.

\section{- Apoyos brindados por la institución} educativa. Valoran el apoyo recibido en las instituciones durante etapas previas $y$ en la actualidad.

"Los profesores nos comprenden, pero uno tiene que estudiar; ponerse las pilas y sí, hay que dormirse tarde y cosas así" (Karina).

"Incluso, yo me iba a salir del colegio y ella [orientadora] me dijo: ¡No! (...) ; si son tres meses lo que le hacen falta a usted!" (Luis).

- Apoyos brindados por personas significativas. Han recibido la solidaridad de seres allegados (familiares, parejas y amistades), dentro de quienes se hace especial mención de las abuelas y los abuelos.

"Mi mamá y mi abuelito son una fortaleza para yo decir 'quiero superarme; quiero salir adelante' (...). Son las personas que han estado ahí en todo momento" (Juan).
"Y gracias a mi abuela, la que nos ha ayudado a nosotros, hemos salido adelante con los estudios; e igual, yo le ayudo cuando trabajo; entonces sí" (Jordan).

Ivannia valora los aportes que le hacen las personas adultas mayores: "Me encanta hablar con personas mayores (...) porque ellos están llenos de sabiduría; le enseñan a uno y si hay algo en esta vida es aprender a tomar consejos; a tomar consejos sabios".

\section{d. Limitaciones}

Las personas participantes no contaron con los apoyos requeridos cuando enfrentaron situaciones que corresponden a los subtemas: condición económica; factores que influyen en la salida anticipada de la educación secundaria; efectos de situaciones familiares de conflicto, enfermedad, adicción o pérdida; y opresión y falta de oportunidad. Adicionalmente, brindan sugerencias con miras al favorecimiento del bienestar y el desarrollo en la actualidad.

- Condición económica. La carencia de recursos económicos ha sido uno de los obstáculos principales.

"Me ha costado mucho porque la hemos pasado difícil (...). Hemos tenido días en que no hay qué comer" (Juan).

"Yo me acuerdo que tenía que ir al colegio con sandalias porque no tenía plata para nada (...). Y he tenido que dejar de lado el estudio para poder trabajar (María Fernanda). 
- Factores que influyen en la salida anticipada de la educación secundaria. De los relatos se extrae que carecieron de algunos de los apoyos que previenen la exclusión educativa de los colegios diurnos.

Los apoyos que no recibieron fueron los relativos a la condición económica, al manejo de la propia conducta, al enfrentamiento de la presión de grupo, al bajo rendimiento y a la repitencia.

Luis relata que se salió del colegio diurno porque "me costaba demasiado (...). Yo soy muy inquieto, profe (...). Nunca se me va a olvidar: había una profesora que no era santo de mi devoción, entonces cada vez que esa profesora daba clases, yo no la dejaba”.

Karina optó por una modalid educativa en la que no tuvo éxito: "Quería sacar Bachillerato por madurez, pero no podía. Sí, matriculaba y estudiaba; y siempre iba hacer los exámenes, pero nunca pasaba".

"Diay, un grupo de amigos, me estaban tratando de convencer de muchas cosas que por dicha no hice, porque yo sé que estaban mal" (Guerin).

- Situaciones familiares de conflicto, enfermedad, adicción o pérdida. En muchos de los casos, cuando se presentaron serias dificultades en sus familias, no contaron con los apoyos requeridos.

"No eran en sí los problemas, porque él se haya ido o cosas así. Tal vez sí, para mi hermano $y$ para mí, era interno" (Guerin).
"Yo no tuve ese acompañamiento tan íntimo, con esas personas que me hubiesen guiado y abierto los ojos (...). Al salir [del hogar del Patronato Nacional de la Infancia] sabía que no tenía personas que me pudieran apoyar" (Greivin).

- Opresión y falta de oportunidad. Algunas personas participantes sufrieron de arbitrariedades durante sus etapas previas. Asimismo, tuvieron insuficientes oportunidades de participación en actividades promotoras de su desarrollo integral.

"Mi papá me sacó del colegio porque él decía que eso era una vagabundería (...). No me dejaron ir más al colegio (...). Y diay, me quedé en la casa haciendo nada (...) hasta que tuve la oportunidad a los 22 años" (Daniela).

Juan considera que no le pusieron atención al deseo que expresaba de niño de tener la oportunidad de practicar regularmente un deporte. Esta carencia ahora le ayuda a definir formas en que a él le gustaría aportar: "en hacer muchos parques recreativos; eso ayuda mucho a la sociedad (...). Ya, en que no sean barrios llenos de drogas, sino barrios llenos de parques, de plazas; ya, porque el deporte es lo que ayuda a la persona a salir adelante".

Cuando era adolescente, María Fernanda deseaba tener la oportunidad de practicar danza e involucrarse en otras actividades que le generaban gran interés: "Yo digo que tal vez si me hubieran apoyado (...) me hubiera ayudado un montón porque me hubiera ayudado a tener confianza en mí misma (...). En la adolescencia, me pasaba viendo tele". 
- Apoyos que favorecerían su desarrollo y bienestar en la adultez joven. Al referirse a apoyos que serían de beneficio en la actualidad, se centraron en dos aspectos: el manejo de sus emociones, y las oportunidades para establecer conexiones significativas.

A María le gustaría tener mayor comunicación con su padre y contar con espacios más amplios en las citas médicas para aclarar dudas acerca de una condición de salud: "Más atención de mi papá porque digamos, a veces él está trabajando o así y los sábados y los domingos digamos, no habla con nosotros (...) del tratamiento digamos; sí, necesito como más ayuda".

A María Fernanda le gustaría lidiar de mejor forma con su enojo y tristeza: "Sí me gustaría como mejorar mi actitud a veces con la vida, porque aunque sea muy positiva, a veces me derrumbo".

La figura dos sintetiza las perspectivas de las personas participantes acerca del fenómeno de estudio.

\section{Discusión}

A partir del análisis de lo expresado por el estudiantado participante, puede afirmarse que sus fuentes de autorrealización y sus recursos guardan concordancia con los fundamentos del bienestar y la transformación a partir de la adversidad. Son personas que han optado en sus vidas por la construcción de significados, a pesar de las dificultades que han enfrentado, producto en buena medida de condiciones de desigualdad.
Entre los temas y subtemas de autorrealizzción se da una interrelación. Por ejemplo, el optimismo alimenta la motivación por alcanzar metas y enfrentar situaciones difíciles. Igualmente, la elección de los valores que guían sus decisiones y acciones favorece las relaciones interpersonales, que a la vez, representan fortalezas externas. Esas fortalezas externas o apoyos se reciben con gratitud y reciprocidad, lo cual alimenta los otros temas, estableciendo así círculos virtuosos.

La presencia de fortalezas no debe impedir el reconocimiento de los obstáculos. Desde la Educación en general y la Orientación en particular, es necesario fortalecer las estrategias de prevención en la línea de las medios identificados en las investigaciones acerca de la resiliencia (Henderson y Milstein, 2003). Se debe brindar al estudiantado, desde edades tempranas: oportunidades para la participación significativa, expectativas elevadas, afecto y apoyo; vínculos prosociales, límites claros y firmes, y fortalecimiento de habilidades para la vida.

Se agradece a quienes participaron como informantes, ya que al compartir sus experiencias y visiones han aportado de forma significativa al desarrollo de otras personas. Al mismo tiempo, se reconoce el aporte de las orientadoras Laura Álvarez Herrera (Sección Nocturna Colegio Técnico Profesional de Calle Blancos) y Carolina Madrigal Ugalde (Colegio Nocturno de la Unión); así como a Mauro Murillo Garita, por su responsabilidad y dedicación como asistente del proyecto. 


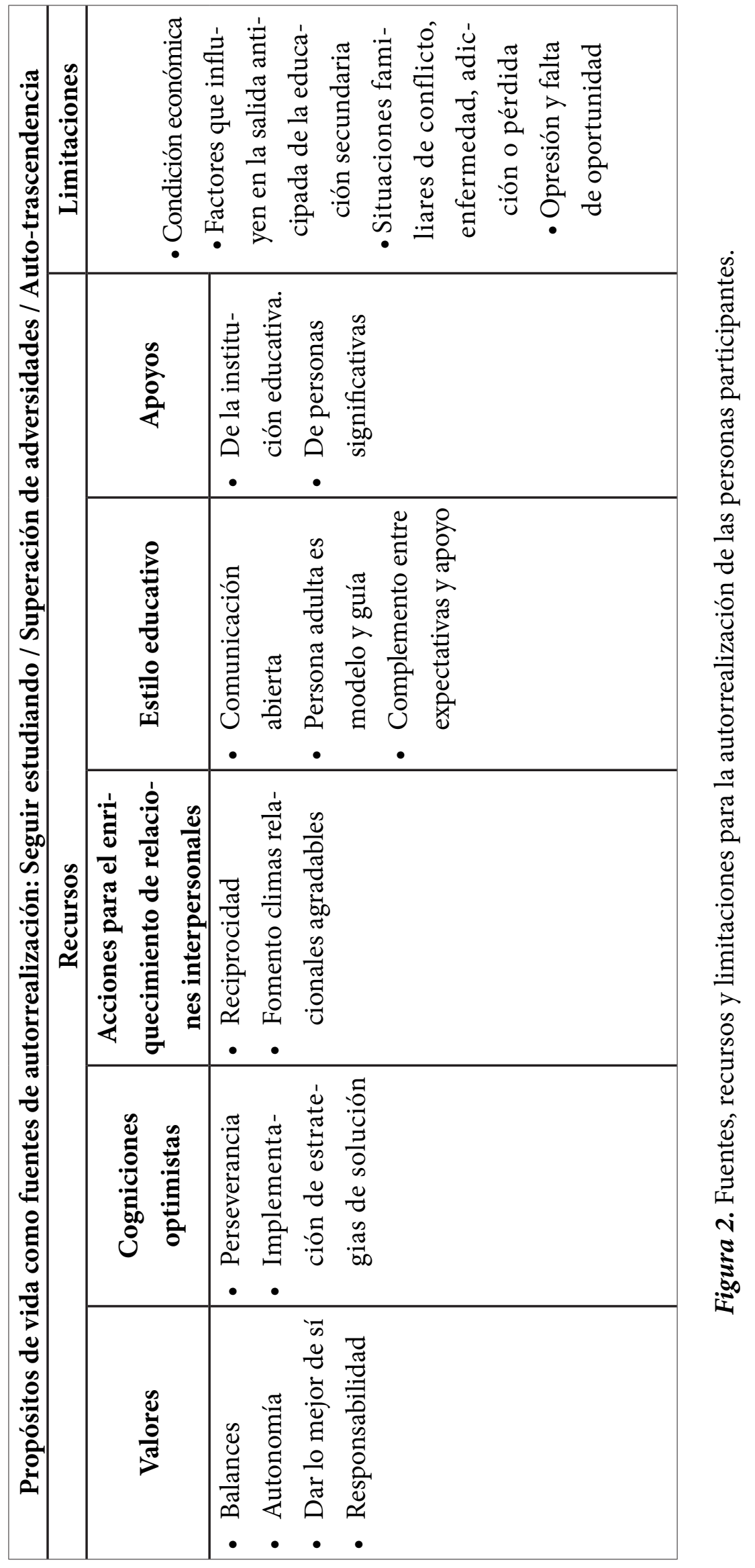




\section{Referencias}

Arguedas, I. (2010). Promoción de la permanencia en la educación secundaria. Manual de temas y estrategias. San José, Costa Rica: Instituto de Investigación en Educación (INIE). Recuperado de http://repositorio.inie.ucr.ac.cr/bitstream/123456789/394/1/permanencia.pdf

Bernal-Guerrero, A. (2002). El concepto de “autorrealización” como identidad personal. Una revisión crítica. Cuestiones pedagógicas: Revista de ciencias de la educación, (16), 11-24. Recuperado de https://idus.us.es/xmlui/bitstream/handle/11441/51291/art_1.pdf

Emmons, R. (2003). Personal goals, life meaning, and virtue: Wellsprings of a positive life. En C. Keyes y J. Haidt. (Editores) Positive Psychology and the Life Well-lived. Washington, DC. American Psychological Association, 105-128. Recuperado de https://www.ncbi.nlm.nih. gov/pmc/articles/PMC4580713/

Erikson, E. (1971). Identidad, juventud y crisis. Buenos Aires: Paidós.

Fiorentino, M. T. (2008). La construcción de la resiliencia en el mejoramiento de la calidad de vida y la salud. Suma Psicológica, 1 (1), 95-114. Recuperado de https://www.redalyc.org/ pdf/1342/134212604004.pdf

Frankl, V. (2012). Psicoterapia y humanismo. ¿Tiene sentido la vida? $8^{\text {a }}$ reimpresión de la 2a edición en español. México: Fondo de Cultura Económica.

Gewirth, A. (1998). Self-fulfillment. Princeton, New Jersey: Princeton University Press.

González, J. (2011). La Orientación en los nuevos escenarios sociales latinoamericanos: Principales retos. Revista Orientación y Desarrollo Humano. Recuperado de http://www.revistaorientacion.com/revista/index.html

Henderson, N. y Milstein, M. (2003). Resiliencia en la escuela. Buenos Aires: Paidós.

Hill, S. y Buss, D. (2008). Evolution and subjective well-being. En M. Eid y R. Larsen (Editores) The Science of Subjective Well-Being. New York: The Guilford Press, 62-79.

Jiménez, E. (2015). Aportes del enfoque de desarrollo humano a la Orientación. En A. Mata (Editora) El desarrollo teórico de la Orientación. Un aporte de la Universidad de Costa Rica. San José, Costa Rica: Universidad de Costa Rica, 115-156. Recuperado de http://inie.ucr. ac.cr/component/booklibrary/194/view/53/Ediciones\%20Digitales/49/el-desarrollo-teorico-de-la-orientacion

Kvale, S. (2011). Las entrevistas en investigación cualitativa. Madrid: Morata. 
Maguire, M. y Delahunt, B. (2017). Doing a thematic analysis: A practical, step-by-step guide for learning and teaching scholars. Ireland Journal of Teaching and Learning in Higher Education (AISHE-J),8 (3), 3351-33514. Recuperado de http://ojs.aishe.org/index.php/aishe-j/ article/viewFile/335/553

Martínez, E. (2013). Los recursos noéticos en la psicoterapia centrada en el sentido. En E. Martínez (Compilador). Manual de psicoterapia con enfoque logoterapéutico. Bogotá: Manual Moderno, S. A.

Miller, R. y Taylor, D. (2016). Does adlerian theory stand the test of time?: Examining individual psychology from a neuroscience perspective. Journal of Humanistic Counseling, 55(2), 111-128. Recuperado de https://onlinelibrary.wiley.com/doi/epdf/10.1002/johc.12028

Rabazo, M. J. (1999). Interacción familiar, competencia socio-escolar y comportamiento disocial en adolescentes (Tesis doctoral). Universidad de Extremadura. Cáceres, España. Recuperado de Dialnet-IntegracionFamiliarCompetenciaSocioescolarYComport-337.pdf

Rodríguez, G., Gil, J. y García, E. (1999). Metodología de la investigación cualitativa. 2a edición. Málaga: Ediciones Aljibe, S. L.

Ryff, C. (2013). Eudaimonic well-being and health: Mapping the consequences of self-realization. En A. S. Waterman (Editor) The best within us: Positive psychology perspectives on eudaimonia. Washington, DC: American Psychological Association, 77-98. Recuperado de http://aging.wisc.edu/pdfs/4326.pdf

Savickas, M., Nota, L., Rossier, J., Dauwalder, J. P., Duarte, M. E., Guichard, J. ... van Vianen, A. (2009). Life designing: A paradigm for career construction in the 21st century. Journal of Vocational Behavior, 75(3), 239-250. Recuperado de http://dare.uva.nl/search?metis.record. $\mathrm{id}=310466$

Scales, P. y Leffert, N. (2004). Developmental Assets. A Synthesis of the Scientific Research on Adolescent Development. Minneapolis: Search Institute.

Seligman, M., Reivich, K., Jaycox, L. y Gillham, J. (2004). Niños optimistas: cómo prevenir la depresión en la infancia. Barcelona: Grijalbo.

Seligman, M. (2011). La auténtica felicidad. Barcelona: Ediciones B, S. A.

Southwick, S. y Charney, D. (2012). Resilience. The science of mastering life's greatest challenges. New York: Cambridge University Press. 
Taff, L. (1966). Values and the school counselor. The High School Journal, 49(5), 214-217. Recuperado de https://www.jstor.org/stable/40366040?seq=1\#metadata_info_tab_contents

Webb, L. (2013). Resilience. How to cope when everything around you keeps changing. West Sussex, Reino Unido: Capstones Publishing Ltd.

Witmer, J. M. y Sweeney, T. (1992). A holistic model for wellness and prevention over the life span. Journal of Counseling and Development, 71(2), 140-148. Recuperado de https://www. jstor.org/stable/40366040?seq=1\#metadata_info_tab_contents

Wong, P. (2012). From Logotherapy to Meaning centered counseling and therapy. En P. Wong (Editor) The Human Quest for Meaning. Theories, Research, and Applications. 2nd ed. (pp. 619-647).

Wong, Y. J. (2015). The psychology of encouragement: Theory, research, and applications. The Counseling Psychologist, 43(2), 178-216. Recuperado de https://www.apa.org/education/ce/ psychology-encouragement.pdf 